\title{
The ionospheric heating beneath the magnetospheric cleft revisited
}

\author{
G. W. Prölss \\ Institut für Astrophysik und Extraterrestrische Forschung, Universität Bonn, Auf dem Hügel 71, 53121 Bonn, Germany \\ Received: 10 September 2004 - Revised: 9 December 2004 - Accepted: 15 December 2004 - Published: 30 March 2005
}

\begin{abstract}
A prominent peak in the electron temperature of the topside ionosphere is observed beneath the magnetospheric cleft. The present study uses DE-2 data obtained in the Northern Winter Hemisphere to investigate this phenomenon. First, the dependence of the location and magnitude of the temperature peak on the magnetic activity is determined. Next, using a superposed epoch analysis, the mean latitudinal profile of the temperature enhancement is derived. The results of the present study are compared primarily with those obtained by Titheridge (1976), but also with more recent observations and theoretical predictions.
\end{abstract}

Keywords. Ionosphere (Polar ionosphere; Plasma temperature and density) - Magnetospheric physics (Magnetosphereionosphere interactions)

\section{Introduction}

One of the most striking features of the dayside polar ionosphere is the sudden increase in the electron temperature beneath the magnetospheric cleft. The first to study this heating effect was Titheridge (1976). Using data derived from Alouette-1 top side ionograms, he presented mean latitudinal profiles of the temperature enhancement and investigated their dependence on magnetic activity and interplanetary conditions. Evidently, the scientific community was so impressed by this study that it did not take up the subject again and/or discontinued work in the field. As a consequence both the effect itself and especially the paper by Titheridge almost fell into oblivion. This prompted the author to repeat the study of Titheridge, however, this time using DE-2 data with their greatly improved spatial resolution. This higher resolution also allowed for more refined analyzing methods, and these are described in the following sections.

\section{Data selection and processing}

A general description of the DE-2 satellite was given by Hoffman and Schmerling (1981), and details on the

Correspondence to: G. W. Prölss

(gproelss@astro.uni-bonn.de)
Langmuir probe experiment can be found in Krehbiel et al. (1981). First electron temperature and density measurements obtained by this instrument were discussed in Brace et al. (1982).

In order to extract the cleft effect on the electron temperature from the unified abstract file of the DE-2 data set, all orbits were divided into four segments, each of which extended from equatorial to polar latitudes. Of these data segments only those obtained in the Northern Hemisphere within \pm 45 days of the December solstice and within the 09:00-15:00 h magnetic local time sector were retained. This is because in the Titheridge study most of the data refer to the same conditions. The selection procedure resulted in a total of 188 orbital segments with sufficient data coverage. For each of these segments, the electron temperature was plotted as a function of invariant latitude and displayed on a terminal screen. As it turned out, clear cleft signatures were observed in nearly all of these temperature profiles, testifying to the quasi-permanent presence of this feature. The terminal plots were also used to roughly parameterize the heating effect. Thus, the latitudes of the equatorward boundary and the maximum of the temperature enhancement, together with their respective temperature values, were determined; see Fig. 1. Since the poleward boundary was often poorly defined, this was not considered. Altogether, 179 temperature profiles and their associated parameter sets are analyzed in the following study.

\section{Location and magnitude of the temperature enhance- ment as functions of magnetic activity}

As first documented by Titheridge (op.cit.), the electron temperature enhancement beneath the magnetospheric cleft shifts towards lower latitudes during disturbed conditions. Here we present an improved description of this effect. In Fig. 2 we plotted the latitude of the temperature maximum as a function of the hourly averaged $A E$ index. As can be seen, the correlation between these two parameters is good, with the correlation coefficient reaching a value of $|r|=0.85$. Also shown is a straight-line approximation fitted to the data (solid line). This is to be compared with the respective regression line for the equatorward boundary of the temperature enhancement which is displaced by about $3.5^{\circ}$ toward lower 


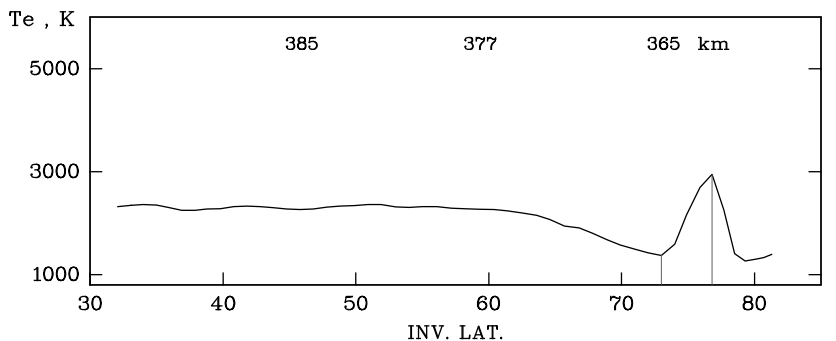

Fig. 1. Example of an electron temperature enhancement in the dayside polar region. Shown are data obtained by the DE-2 satellite on 18 January 1983 in the Northern Hemisphere. The measurements refer to a magnetic local time of approximately $13: 10 \mathrm{~h}$ and to moderate magnetic activity. The observation heights are indicated in the upper part of the figure. Vertical lines mark the equatorward boundary and the maximum of the temperature increase.

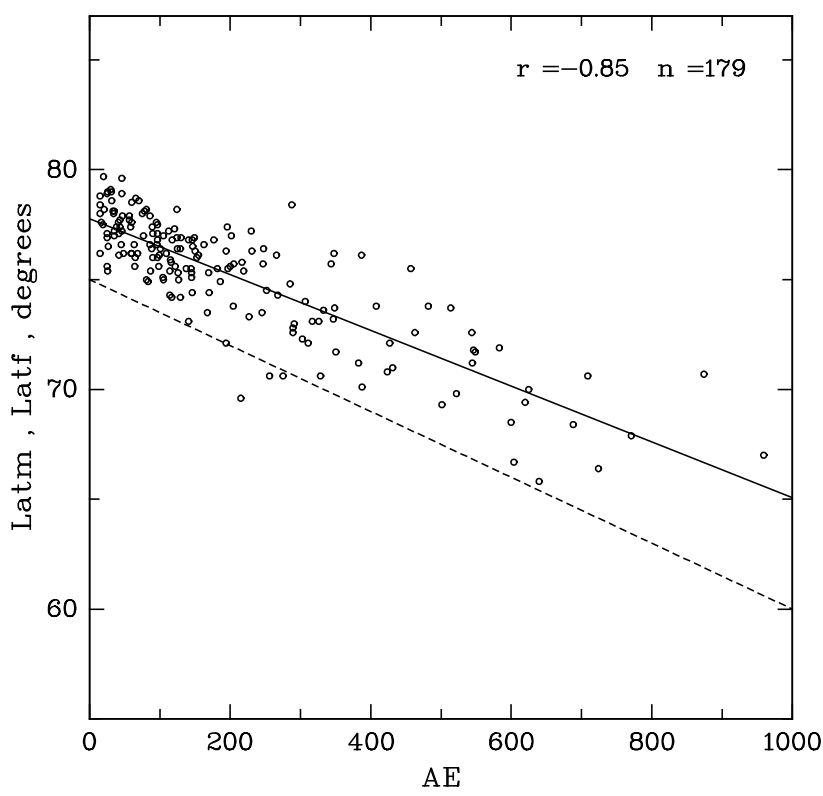

Fig. 2. Changes in the position of the temperature peak with the level of magnetic activity. The scatter plot shows the invariant latitude of the maximum of the temperature enhancement Latm as a function of the hourly averaged $A E$ index. A straight line has been fitted to the data: Latm $[\mathrm{deg}]=77.8-0.013 \cdot A E$ (solid line). The regression coefficient reaches 0.85 and the total number of data points is 179. Also shown is the regression line for the invariant latitude of the equatorward boundary of the temperature increase: Lat $[\mathrm{deg}]=75.0-0.015 \cdot A E$ (dashed line).

latitudes (dashed line). Extrapolating both lines to $A E=0$, we find that during magnetically quiet conditions the equatorward boundary and the temperature maximum should be located at about $75^{\circ}$ and $78^{\circ}$ invariant latitude, respectively.

The latitude of the temperature maximum was also correlated with other magnetic activity indices like the $K_{p}, a_{p}$ and $D_{s t}$ indices, partly including the temporal development of these indices. As it worked out, these correlations were not as good as for the $A E$ index. A similarly high correlation coeffi-

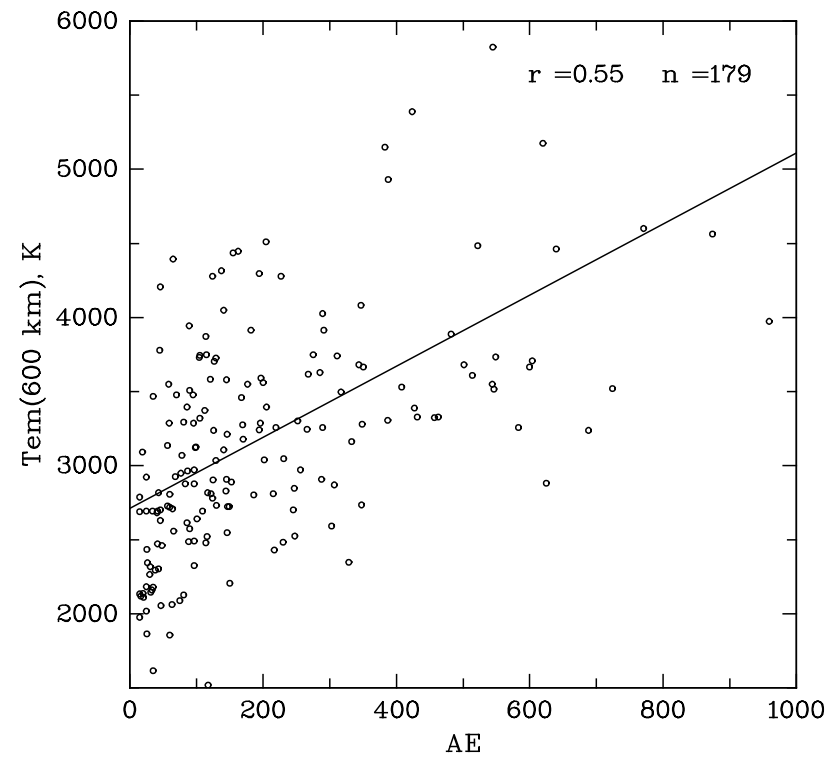

Fig. 3. Changes in the magnitude of the temperature peak with the level of magnetic activity. Shown is a scatter plot of the maximum temperatures $T \mathrm{em}$ as a function of the hourly averaged $A E$ index. These temperatures have been adjusted to a common altitude of $600 \mathrm{~km}$. Also shown is the respective regression line: $\operatorname{Tem}[\mathrm{K}]=2712+2.4 \cdot A E$.

cient was obtained only for the negative $B_{z}$ component of the interplanetary magnetic field. We also checked Titheridge's suggestion that the location of the temperature enhancement depends on the sector polarity of the interplanetary magnetic field. No correlation of this kind was observed.

Finally, the dependence of the magnitude of the temperature increase on the level of magnetic activity was investigated. To this end the peak temperature was plotted as a function of the $A E$ index; see Fig. 3. As can be seen, the scatter of the data points is quite large and the correlation coefficient correspondingly low $(r=0.55)$. There is, however, a general trend for the heating effects to become larger with increasing magnetic activity. A somewhat better correlation was obtained when the $A E$ index was replaced by the dynamic pressure of the solar wind or the product of these quantities.

\section{Mean latitudinal profile}

As illustrated in Fig. 1, the temperature enhancement in the cleft region is confined to a relatively narrow latitudinal range. Also, its position is constantly changing in response to changes in geomagnetic activity. Therefore, special care must be exercised when deriving the mean latitudinal profile of this phenomenon. Here the following procedure was adopted.

In a first step all data were adjusted to a common altitude of $600 \mathrm{~km}$. This was done because the electron temperature depends on altitude both inside and outside the heating region (see again Titheridge, op.cit.). A linear regression 
analysis was performed to estimate the temperature gradient in the topside ionosphere. For the data set at hand, this ranged between about 2 and $3 \mathrm{~K} / \mathrm{km}$. To simplify matters, a constant temperature gradient of $2.5 \mathrm{~K} / \mathrm{km}$ was used for the height adjustment.

In a second step all available latitudinal profiles (including that shown in Fig. 1) were superimposed in such a way that the maxima of the temperature enhancements were aligned and located at a common reference latitude (superposed epoch analysis). This way the basic latitudinal structure of the temperature enhancement was kept intact and not smeared out by the averaging procedure.

In a third step the median and the upper and lower quartiles of the temperature values were determined for each latitude. Finally, spline curves were fitted to these parameters. The end product of this procedure is shown in Fig. 4. Here the latitude coordinates refer to an $A E$ index of 200. This axis should be shifted to the left or right depending on the respective level of magnetic activity.

As is evident from Fig. 4, the mean electron temperature enhancement in the cleft region stands out as an isolated narrow peak similar to that shown in Fig. 1. The temperature increase is of the order of $1200 \mathrm{~K}$, which corresponds to a relative increase of about $60 \%$. At the same time, the full width of the temperature enhancement is about $6^{\circ}$. These properties should be compared with those derived by Titheridge (op.cit.), whose mean latitudinal profile is also shown (dashed line; note that this profile was obtained by interpolating his profiles for 400 and $1000 \mathrm{~km}$ altitude). As is evident, the mean temperature increase derived by this author is considerably smaller and much wider. This is not surprising considering the relatively crude latitudinal resolution of the data set at his disposal and the averaging procedure employed.

How does the electron density behave in the neighborhood of the temperature peak? To answer this question, mean latitudinal profiles of the electron density were generated, again using the latitude of the peak electron temperature as a common reference location. We find that on the average a slight decrease in the electron density is observed near the temperature enhancement; see Fig. 5. If individual profiles are examined, both increases and trough-like decreases in the electron density are observed, but most of the time these are not strictly co-located with the temperature enhancement.

\section{Discussion}

The present study provides an improved description of the ionospheric heating effect beneath the magnetospheric cleft. This should prove useful in several respects.

First, such a description may be incorporated into empirical models of the ionosphere. The International Reference Ionosphere, for example, does not contain a specification of this phenomenon (Bilitza, 2001).

Second, the derived heating effect should be compared with the results of theoretical studies. Take, for example,

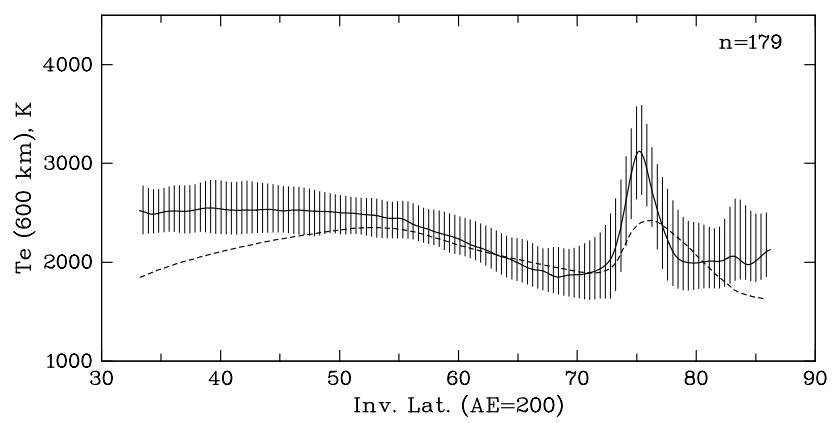

Fig. 4. Mean latitudinal profile of the electron temperature enhancement in the dayside polar region in winter. Plotted is the median of 179 temperature profiles which have been superimposed in such a way that the latitude of the temperature peak serves as a common reference location. The shaded area indicates the range between the lower and upper quartiles. The specific latitudes indicated on the abscissa apply for an hourly averaged $A E$ index of 200 (see Fig. 2). Also shown is the mean latitudinal profile derived by Titheridge (1976) for similar conditions (dashed line). All temperatures refer to a common altitude of $600 \mathrm{~km}$.

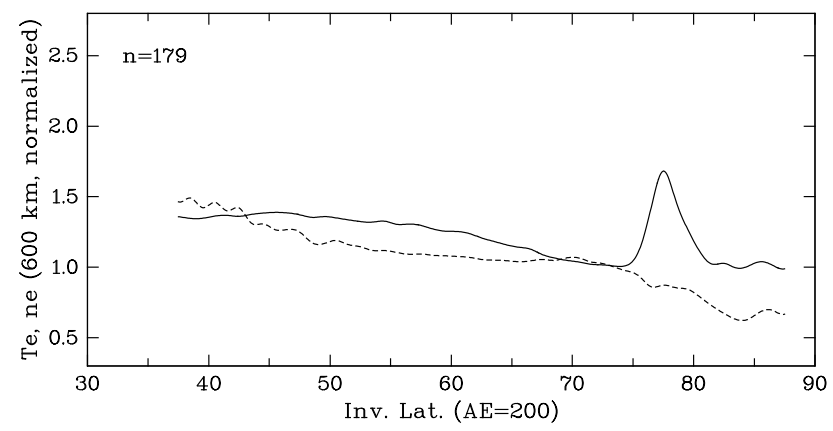

Fig. 5. Comparison of the mean latitudinal profiles of electron temperature (solid line) and electron density (dashed line) in the dayside polar region in winter. The format of data presentation is similiar to that used in Fig. 4, except that in addition to the height adjustment, the data have also been normalized. Here the temperature and density recorded at the equatorward boundary of the temperature peak (i.e. at Latf) served as reference values.

the recent simulation study by Vontrat-Reberac et al. (2001). These authors find that immediately after turning on the cusp precipitation in their ionospheric model, the electron temperature in the topside ionosphere increases by about $1000 \mathrm{~K}$, which is close to what is actually observed on the average. Also, the lack of a clear signature of the cleft precipitation on the electron density seems to be understood (e.g. Pitout and Blelly, 2003).

Third, our results may be compared with other observations. These include electron temperature enhancements in the cleft region as measured by incoherent backscatter facilities (e.g. Doe et al., 2001); dayside auroral emissions at $630 \mathrm{~nm}$ produced by the increase in the electron temperature as recorded by ground-based photometers (e.g. Sandholt et al., 2002); and satellite-based measurements of the 
particle precipitation in the cleft region (e.g. Carbary and Meng, 1986). The latter study, for example, specifies the equatorward shift of the cleft precipitation with increasing magnetic activity. They find that for moderate magnetic activity $(A E(1 \mathrm{~min})=200)$, the center of the precipitation region is located near $76^{\circ}$, which is close to where the temperature peak is observed in the present study for similar conditions.

Finally, the close connection between ionospheric and thermospheric heating effects should be pointed out. Whereas the lower thermosphere beneath the cleft region is primarily heated by ionospheric currents (e.g. Prölss, 1981; Lühr et al., 2004), the upper thermosphere is also heated by the hot electron gas. This thermal coupling deserves further study.

A more detailed comparison with theory and other observations has been postponed until a larger data set is available. This extended data set will include measurements obtained during all seasons and in both hemispheres.

Acknowledgements. The DE-2 data used in this study were kindly provided by the NASA National Space Science Data Center. I am grateful to all the experimenters who contributed to this data set. I am also indebted to Ben Bekhti, N., Schrüfer, K., and Winkel, B. for their help in preparing this manuscript.

Topical Editor T. Pulkkinen thanks J. E. Titheridge for his help in evaluating this paper.

\section{References}

Bilitza, D.: International Reference Ionosphere 2000, Radio Sci., 36, 261-275, 2001.

Brace, L. H., Theis, R. F., and Hoegy, W. R.: A global view of Fregion electron density and temperature at solar maximum, Geophys. Res. Lett., 9, 989-992, 1982.

Carbary, J. F. and Meng, C. I.: Correlation of cusp latitude with $B_{z}$ and $A E(12)$ using nearly one year's data, J. Geophys. Res., 91, $10047-10054,1986$.

Doe, R. A., Kelly, J. D., and Sánchez, E. R.: Observations of persistent dayside $\mathrm{F}$ region electron temperature enhancements associated with soft magnetosheathlike precipitation, J. Geophys. Res., 106, 3615-3630, 2001.

Hoffman, R. A. and Schmerling, E. R.: Dynamics Explorer program: An overview, Spaces Sci. Instr., 5, 345-348, 1981.

Krehbiel, J. P., Brace, L. H., Theis, R. F., Pinkus, W. H., and Kaplan, R. B.: The Dynamics Explorer Langmuir probe instrument, Space Sci. Instr., 5, 493-502, 1981.

Lühr, H., Rother, M., Köhler, W., Ritter, P., and Grunwaldt, L.: Thermospheric up-welling in the cusp region, evidence from CHAMP observations, Geophys. Res. Lett., 31(L06905), doi:10.1029/2003GL019314, 2004.

Pitout, F. and Blelly, P.-L.: Electron density in the cusp ionosphere: increase or depletion?, Geophys. Res. Lett., 30(14), doi:10.1029/2003GL017151, 2003.

Prölss, G. W.: Latitudinal structure and extension of the polar atmospheric disturbance, J. Geophys. Res., 86, 2385-2396, 1981.

Sandholt, P. E., Carlson, H. C., and Egeland, A.: Dayside and polar cap aurora, Kluwer Acad. Publ., Dordrecht, 2002.

Titheridge, J. E.: Ionospheric heating beneath the magnetospheric cleft, J. Geophys. Res., 81, 3221-3226, 1976.

Vontrat-Reberac, A., Fontaine, D., Blelly, P.-L., and Galand, M.: Theoretical predictions of the effect of cusp and dayside precipitation on the polar ionosphere, J. Geophys. Res, 106, $28857-$ $28865,2001$. 